\title{
Validation of a set of stimuli to investigate the effect of attributional processes on social motivation in within-subject experiments
}

\author{
Fucci E. ${ }^{1}$, Baquedano C. ${ }^{2}$, Abdoun $0 .{ }^{2}$, Deroche J. ${ }^{3}$, Lutz A. ${ }^{2}$
}

1. Institute for Globally Distributed Open Research and Education (IGDORE)

2. Lyon Neuroscience Research Center, DYCOG Team, INSERM U1028 - CNRS UMR5292, Lyon, France 3. Institut des Sciences Cognitives Marc Jeannerod, CNRS UMR 5304, Lyon, France

Address correspondence to:

Enrico Fucci, Institute for Globally Distributed Open Research and Education (IGDORE, https://igdore.org/). E-mail: enrico.fucci@igdore.org 


\section{Abstract}

Attribution of responsibility for the causes of suffering is one of the main factors that influence responses to individuals in distress. While the role of attributional processes on prosocial motivation has been widely investigated in social psychology, only few attempts have been made to characterize their behavioural and neurophysiological underpinnings. This is partly due to the lack of stimuli that can facilitate within-subject experimental designs. To overcome this problem, we created a set of stimuli consisting of videos depicting people in different situations of distress. Each video is paired with short stories that aim to manipulate the perceived degree of responsibility of the main character.

To validate the stimuli, we investigated the effect of different context-video pairs on self-report measures of participants' subjective experience. We found that different contexts preceding the same video can influence blame and responsibility judgments, affective responses and willingness to help. In a complementary analysis, we replicated previous findings on the influence of empathy and responsibility on willingness to help, showing how the latter is mediated by moral judgments.

Finally, we observed a general increase in responses times when videos were paired with responsible contexts. We provide interpretations of this finding that can relate attribution accounts to prominent theories in moral psychology.

Overall, this study highlights the possibility of expanding existing theories on prosocial motivation by implementing a set of stimuli that includes multiple scenarios and allows for the collection of third person measures in within-subject designs. 


\section{Introduction}

Within evolutionary analysis, blameworthiness of the sufferer is one of the main factors that modulate the observer's reaction to others' suffering (Goetz et al., 2010). In particular, the degree to which a person is perceived as being responsible for their suffering is an important mediator of blame attribution, affective responses and willingness to help. This effect has been widely investigated over the last decades by attributional accounts of social behaviours such as help giving and aggression (Weiner, 2006). According to attribution theory, judgments of responsibility influence the emotional reaction toward a person in need (e.g. eliciting sympathy or anger). This will, in turn, affect helping intentions and behavioural responses. Weiner's theory is supported by empirical evidence showing that when a person in need is perceived as having control over the causes of their condition, this in turn elicits less sympathy and more anger in the observer, resulting in less willingness to help compared to a situation when the person is perceived as a victim of external causes (see Rudolph et al., 2004 for a meta-analysis of 39 studies on helping intentions). Characterizing the role of attributional processes in prosocial behaviours does not only help us to understand fundamental dynamics of human interactions but can have a tremendous impact on the way we deal with suffering and stigmas in modern societies, guiding policy making in social and health care. For instance, attribution of responsibility can influence the disposition of healthcare professionals towards patients (Philip et al., 2014), the reactions to victims of cyberbullying (Weber et al., 2013) and the seeking of professional help in individuals affected by mental health problems (Mojtabai, 2010; Vogel et al., 2007).

Within this research domain, models of attribution lay heavily on evidence derived from subjective measures and cross-sectional experiments. The study of behavioural and neurophysiological mechanisms underlying attributional processes is currently in an early stage, despite its potential to expand existing psychological models as well as to shed further light over the biological grounds of the interplay between affect and cognition. 
To our knowledge, only few attempts have been made to characterize the effect of responsibility attribution on neural dynamics and autonomic activity (e.g. Azevedo et al., 2014; Decety et al., 2009; Krendl et al., 2013) and no studies have implemented implicit behavioural measures. While this research provides evidence supporting the role of perceived responsibility in modulating biological responses to suffering, it is affected by several methodological limitations. First, only specific situations and targeted groups are considered, with a focus on stigmatized individuals. Second, whether consisting of pictures or short videos, the experimental stimuli do not control for general features such as arousal and valence (except in Decety et al., 2009).

The remaining literature on attributional processes share some of these limitations regarding experimental stimuli, as well as being affected by other issues. As previously stated, most of the studies are based on subjective measures of emotional responses, whether to short stories (e.g. Kogut, 2011), vignette scenarios (e.g. Seacat et al., 2007; Philip et al., 2014) or imaginary events (e.g. Halkjelsvik \& Rise, 2014). More importantly, none of the studies that were included in Rudolph et al. (2004) meta-analysis or that were published later implemented a set of stimuli that allowed for a repeated-measures approach (except for those using neuroimaging techniques). Consequently, and like in most psychological research, theoretical models of attributional processes are supported almost exclusively by cross-sectional data but commonly interpreted as personal level processes. This passing from cross-sectional data to within-person processes implicitly assumes that the structure of interindividual differences reflects intraindividual processes. However, there are many situations where this is simply not true (Hamaker, 2012). ${ }^{1}$

\footnotetext{
${ }^{1}$ Hamaker discusses this issue extensively and provides a simple example to illustrate potential discrepancies between levels of analysis. Usually, when one tries to type faster, it is at the expense of accuracy and vice-versa: this is but one instance of the ubiquitous speed-accuracy trade-off phenomenon. Therefore, typing speed and accuracy are negatively correlated at the individual level. However, training in typing may improve both speed and accuracy such that people who are skilled at typing are both faster and more accurate: at the population level, typing speed and accuracy appear positively correlated. Discrepancies between levels of analysis in directions of association are at the source of the notorious Simpson paradox.
} 
To overcome methodological limitations and facilitate research in this field using third person methods, we created a set of stimuli that allow for the manipulation of perceived responsibility in different scenarios of suffering. Specifically, we selected a subset of emotional and neutral videos from the Socio-affective Video Task (SoVT; Klimecki et al., 2013), which were previously validated and matched in terms of valence, arousal and empathy elicitation. Emotional videos depict distressed people in different situations, while neutral videos show people engaged in everyday activities. We created pairs of short stories (contexts) associated with each video. In emotional videos, the contexts aim at manipulating the perceived responsibility of the main character/s for being in their condition (see the Methods section for details on the criteria used to select videos and create short stories). The set of stimuli was created with the general objective of allowing its implementation in within-subjects, repeated measures experimental designs. We used video stimuli to elicit more robust emotional responses compared to vignettes and short stories commonly implemented in this research field (Grühn \& Sharifian, 2016).

In the present study, we sought to validate the new set of stimuli in terms of their capacity to manipulate perceived responsibility and blameworthiness depending on how different contexts were associated to the same video in each context-video pair. We also hypothesized that contexts describing a character as being responsible for their condition would result in lower participants' empathy and willingness to help compared to when the same character is described as not responsible.

Additionally, we explored the within-subject associations between perceived responsibility, blame, empathy and willingness to help and compared them to results observed in previous empirical studies of interindividual differences (e.g. Halkjelsvik and Rise, 2014; Kogut, 2011; Rudolph et al., 2004).

Finally, we sought to highlight how using these stimuli in repeated measures experiments can facilitate the study of attributional processes using implicit measures and provide further evidence into their 
behavioural mechanisms. As an example, we conducted exploratory analyses on the effect of different contexts-video pairs on response times.

\section{Methods}

\subsection{Subjects}

Sixty healthy individuals ( $31 \pm 7.6$ years old; 27 females, 32 males [age and sex info are missing for 4 and 1 participant, respectively], all white Caucasians) participated in the study. Participants were recruited in the local community by flyers, ads in social media and word to mouth. Each participant was screened for the following exclusion criteria: history of neurological or psychiatric conditions, severe hearing loss, uncorrected eyesight, use of psychoactive medication, consumption of recreational drugs (including alcohol) the day before participating in the study. They were informed that the paradigm contained emotional stimuli which could impress highly sensitive individuals and that they could end the task at any time. All subjects presented a proof of affiliation to social security, provided signed informed consents and received a monetary compensation for their participation. The study was approved by the appropriate regional ethics committee on Human Research (CPP SudEst IV, 2015-A01472-47).

\subsection{Stimuli}

Video stimuli were derived from a subset of the videos implemented in the Socio-affective Video Task (SoVT; Klimecki et al., 2013). They consisted of emotional videos depicting people in distress and neutral videos of people involved in everyday activities. They were selected from material cast for news and documentaries. The subset of videos used in the present study consisted of 20 emotional and 20 neutral videos. This subset was created by selecting, from the original set of 72 videos used in the SoVT, those emotional videos that would allow a manipulation of the perceived responsibility of the sufferer when preceded by a specific context. To this purpose, we selected videos which did not 
include children as main subjects (although some videos might display children as secondary subjects). For the present study, audio traces were removed from each video.

During the task, each video was introduced by a contextualizing short story. For each video, we created two different short stories. In emotional videos, the two stories aimed at manipulating the degree of responsibility ascribed to the main subject/s for their suffering (responsible and not responsible). Several strategies were used to differentiate Responsible from Not Responsible contexts: some contexts differed in terms of controllability (e.g. a mother who lost her child because of neglecting behaviour compared to a fatal disease), others ascribed different acts to the sufferers (e.g. prisoners convicted for war crimes compared to protesting an authoritarian regime) and others manipulated the identity of the suffer (e.g. a terrorist compared to a victim of terrorism). Other elements of the stories were kept identical (Figure 1 provides an example of contexts used for an emotional video). If a video depicted a single character as the main subject, a proper noun was included in the context and participants were told to answer questions focusing on that particular character. If, instead, a group of characters was the main subject, we specified who they were (e.g. prisoners, workers, villagers) and participants were told that no specific focus was required. The short stories did not contain any reference to real places or people, and their fictional nature was disclosed to the participants at the end of the task. For neutral videos, contexts were created following the same criteria as for the emotional stimuli, but without aiming at manipulating the perceived degree of responsibility of the main character/s (two contexts for each neutral video were implemented only to maintain balance in the experimental design). The contexts were originally written in English and subsequently translated into French for being implemented in the present study. For the French version, audios were also recorded. They consist in a female or male voice reading the context message for all contexts and video types (two audio versions). The set of stimuli is available online, in English and French versions, at https://osf.io/8zytp/. 


\section{(p)) CONTEXT A: Responsible}

Fatima and her family are evicted from a refugee camp after they have been caught stealing prescription drugs from the camp hospital and sell them to drug addicts

\section{4)) CONTEXT B: Not-responsible}

Fatima and her family are evicted from a refugee camp because of lack of receptional capacity

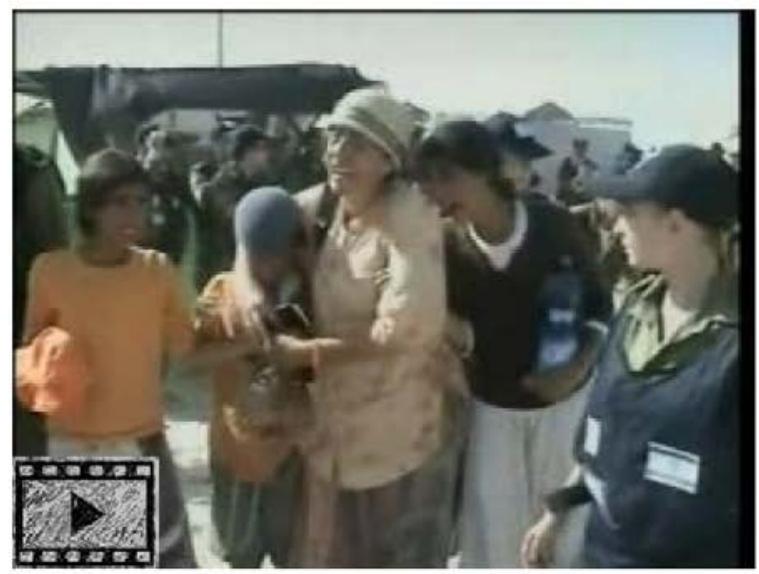

Figure 1. Example of the context-video stimuli created for the present study. For each emotional video, two contexts in the form of short stories were created. Only one context is displayed before each video, resulting in stimuli consisting in different context-video pairs. In this example, two contexts associated to an emotional video are presented, together with a screenshot from the sample video.

\subsection{Paradigm}

Participants sat on a comfortable chair in a quiet room, wearing a pair of headphones. The task ran on a laptop PC and stimuli were presented using PsychoPy version 1.83 (Peirce et al., 2019). The task consisted in the presentation of the forty context-video pairs, each followed by six questions to be answered on a 1 to 9 Likert-like scale using a computer mouse. Participants received oral instructions and underwent two training trials before the start of the experiment, when instructions were repeated on the screen (the material for training trials [two neutral videos and contexts] is available online at https://osf.io/8zytp/). The training trials were implemented to help participants familiarizing with the questions following each context-video pair. The six questions were presented in a fixed order and 
investigated different types of judgments and dimensions of the subjective experience of a participant after watching each context-video pair. Specifically, we asked participants to rate: 1) the valence of the emotional content expressed in the video, 2) the level of emotional arousal in response to the video, 3) the degree of empathy for the main character/s, 4) a judgment on how much the main character/s were responsible of their situation (responsibility), 5) a judgment on how much the main character/s deserved to be in that situation (deservingness) and 6) the participant's willingness to help the main character/s (questions and scales are available online at https://osf.io/8zytp/). To account for the idiosyncrasy of moral judgment in low-emotional videos, subjects were given the possibility to skip to the next question whenever they thought a question was not making sense for the situation at hand (this applied to questions 4, 5 and 6). They skipped to the next question by scoring 5 out of 9 on the scale.

Two subsets of stimuli were created by changing the type of context preceding the videos. Set 1 included half of the emotional videos with responsible and the other half with not-responsible contexts; half of the neutral videos with context A and the other half with context B. Set 2 included the exact same videos, but with inverted context-video associations. Participants were randomly assigned to one of the two sets of stimuli. For each participant, the stimuli presentation order was randomized.

\subsection{Analysis}

All statistical analyses were performed using R (v. 3.5.2, R core team, 2017).

For questions Q4 (responsibility), Q5 (deservingness) and Q6 (willingness to help), the value 5 (corresponding to "not relevant") was replaced by NA (question 4 NAs = 103 for emotional and 578 for neutral videos; question 5 NAs $=130$ for emotional and 747 for neutral videos; question 6 NAs $=175$ for emotional and 641 for neutral videos) and values 6 to 9 were mapped onto the 5-8 range. The analysis of self-reports data for questions on responsibility, deservingness and willingness to help 
was restricted to emotional videos only due to the inapplicability of such questions to neutral videos (a claim supported by the high percentage of missing answers to neutral videos).

\subsubsection{Linear mixed models}

We used linear mixed-effects models (R package Ime4; Bates et al., 2014) to investigate the effects of the experimental manipulation on self-reports and log-transformed response times (RT) separately for each scale. Holm's family-wise error rate controlling procedure was used to account for multiple tests (Holms, 1979).

For questions on valence, arousal and empathy, we fitted models including a three-levels factor (StimType) as fixed effect, corresponding to three stimulus types (emotional videos preceded by responsible context, emotional videos preceded by not-responsible contexts and neutral videos). The same factor was included as by-subject and by-video random slopes. The model fixed effect was estimated using a type III F-test with Satterthwaite's method for approximation of degrees of freedom. Paired t-tests, corrected for multiple comparisons using Tukey honestly significant difference test (HSD), were used as post-hoc tests comparing estimated marginal means using the $R$ package emmeans (Lenth et al., 2020). For questions on responsibility, deservingness and willingness to help, we created models including a two-levels factor (Context) as fixed effect, corresponding to two stimulus types (emotional videos preceded by responsible context and emotional videos preceded by not-responsible context). Context was included as by-subject and by-video random slopes. The effect of Context was assessed with a paired t-test comparing estimated marginal means from the initial model.

For all models, we report effect sizes in the form of difference values and $95 \%$ confidence intervals from contrasts between estimated marginal means. 


\subsubsection{Regularized partial correlation networks}

In order to assess the relationships between the 6 rating scales, we built a regularized partial correlation network based on within-subject variability in the emotional videos and responsible contexts. Data from the not-responsible contexts was excluded to avoid spurious correlations due to a general effect of context on all variables. For each variable, the participant's mean was subtracted in order to remove the between-subject variance. Partial correlations between the 6 centred variables (corresponding to the six self-report scales) were computed. In order to reduce the risk of overfitting without losing power, the resulting network was regularized using the LASSO, yielding a sparse network with many edges exactly set to 0 . Confidence intervals for edge weights and associated $p$ values were estimated using non-parametric bootstrapping with 10,000 bootstraps constructed by resampling with replacement. Edges with $p>.05$ were not retained. All these operations were performed using the qgraph (Epskamp et al., 2012) and bootnet (Epskamp et al., 2018) packages for R.

\subsubsection{Statistical power}

An examination of a selection of studies included in the meta-analysis of Rudolph et al. (2004) revealed that effect sizes of the effect of controllability on attribution of responsibility are generally very high (Cohen's $d>1$ ), while those on willingness to help typically range between 0.6 and 0.9 (for accurate and relevant estimates of effect sizes, we considered studies with large sample sizes and stimuli featuring intense suffering). Our sample size $(\mathrm{N}=60)$ is largely sufficient to detect such effects (see table 7 in Brysbaert 2019). Considering that our participants were presented with 10 observations for each cell (compared to only 1 or 2 in the literature), this power estimation is likely to be conservative. For within-subject correlations, our sample size $(\mathrm{N}=60 \times 10=600)$ is sufficient to detect correlation coefficients as low as 0.12 with $80 \%$ power. 


\section{Results}

\subsection{Effect of experimental manipulation on self-reports}

A spreadsheet including average responses to each video and for each scale is available at https://osf.io/8zytp/. Figure 2 shows data distribution and significant contrasts for each scale.

For questions on valence, arousal and empathy, we found a statistically significant main effect of stimulus type (valence $F(2)=64.9, p<0.001$; arousal $F(2)=125.9, p<0.001$, empathy $F(2)=120.7, p$ $<0.001$.

Post-hoc tests showed that neutral videos, compared to emotional videos preceded by responsible or not-responsible contexts, were judged to have higher valence (emotional_Resp. - neutral: tratio(79.5) $=-11.4, p<0.001$, estimate $[95 \% \mathrm{Cl}]=-2.6[-3.2,-2.1]$; emotional_notResp. - neutral: $\mathrm{t}-$ ratio(79.3) $=-10.6, \mathrm{p}<0.001$, estimate $[95 \% \mathrm{CI}]=-2.9[-3.6,-2.3])$, elicited lower arousal (emotional_Resp. - neutral: t-ratio $(79.5)=10.3, p<0.001$, estimate[95\% Cl] $=2.8[2.1,3.4]$; emotional_notResp. - neutral: t-ratio(62) $=14.8, \mathrm{p}<0.001$, estimate $[95 \% \mathrm{Cl}]=3.5[3,4.1])$ and less empathy (emotional_Resp. - neutral: t-ratio(70.2) $=7.6, \mathrm{p}<0.001$, estimate[95\% Cl] $=2.1[1.4,2.8]$; emotional_notResp. - neutral: t-ratio $(58.9)=14.8, \mathrm{p}<0.001$, estimate $[95 \% \mathrm{Cl}]=3.6[3,4.2])$.

For all questions, we found a statistically significant difference between emotional videos preceded by responsible, compared to not-responsible contexts. Emotional videos preceded by responsible, compared to not-responsible contexts, had more negative valence and elicited lower arousal and less empathy. The videos main character/s were judged as having more responsibility and deservingness for their condition. Finally, participants willingness to help was lower in response to emotional videos preceded by responsible, compared to not-responsible contexts. A summary of the estimates and significance of post-hoc tests for each scale is reported in Table 1. 
Table 1. Summary statistics of pairwise comparisons of estimated marginal means between responsible and not responsible contexts (Emotional_Resp. - Emotional_NotResp) for emotional videos. t-ratios and p values are reported, together with marginal means contrast estimates and $95 \%$ confidence intervals for each response scale.

\begin{tabular}{|l|c|c|c|}
\cline { 2 - 4 } \multicolumn{1}{c|}{} & \multicolumn{3}{c|}{ Emotional_Resp. - Emotional_NotResp } \\
\hline Variable & t-ratio $(d f)$ & $p$ & estimate $(95 \%$ CI) \\
\hline Valence & $2.8(37.4)$ & $\mathbf{0 . 0 2}$ & $0.3(0.03,0.5)$ \\
\hline Arousal & $-5.7(32.5)$ & $<.001$ & $-0.7(-1,-0.4)$ \\
\hline Empathy & $-8(42.7)$ & $<.001$ & $-1.5(-2,-1)$ \\
\hline Responsibility & $13.8(24.1)$ & $<. \mathbf{0 0 1}$ & $4(3.4,4.5)$ \\
\hline Deservingness & $10.1(29.1)$ & $<. \mathbf{0 0 1}$ & $3(2.4,3.6)$ \\
\hline Help & $-9(38.1)$ & $<. \mathbf{0 0 1}$ & $-2.3(-2.8,-1.8)$ \\
\hline
\end{tabular}


茞Emotional_Resp 置Emotional_NotResp 置Neutral

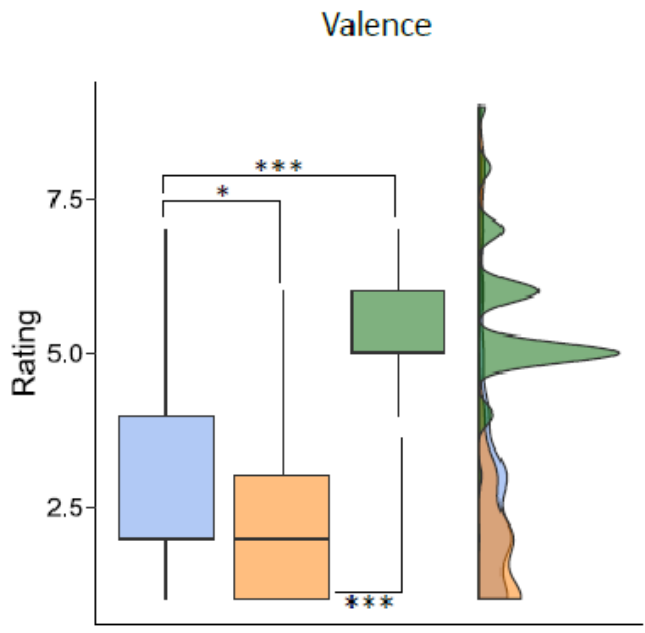

Responsibility

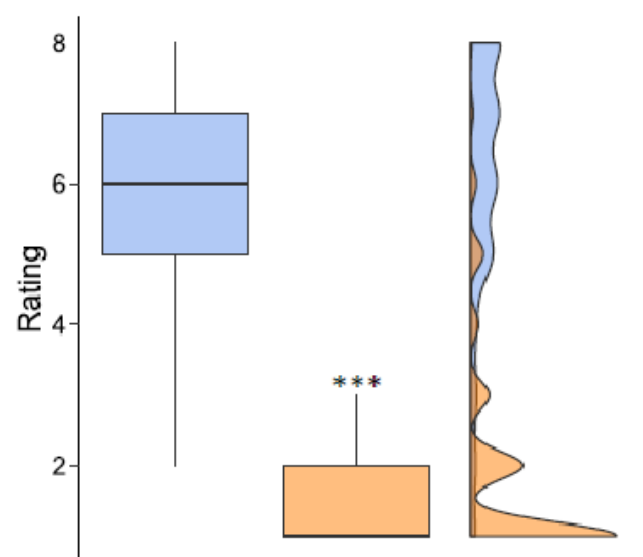

Arousal

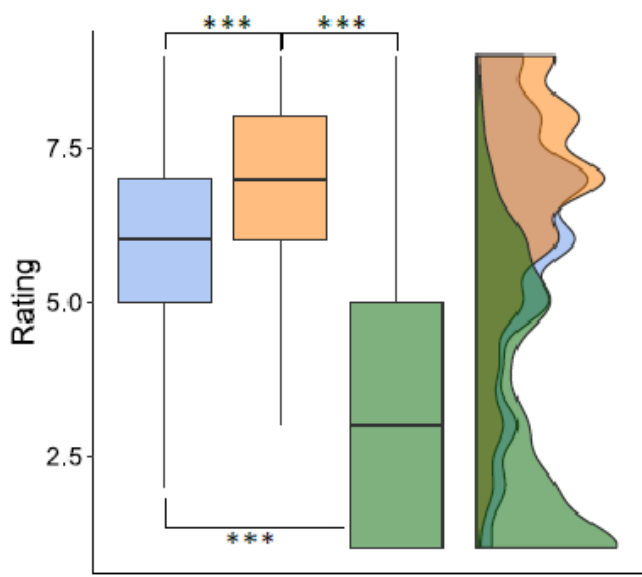

Deservingness

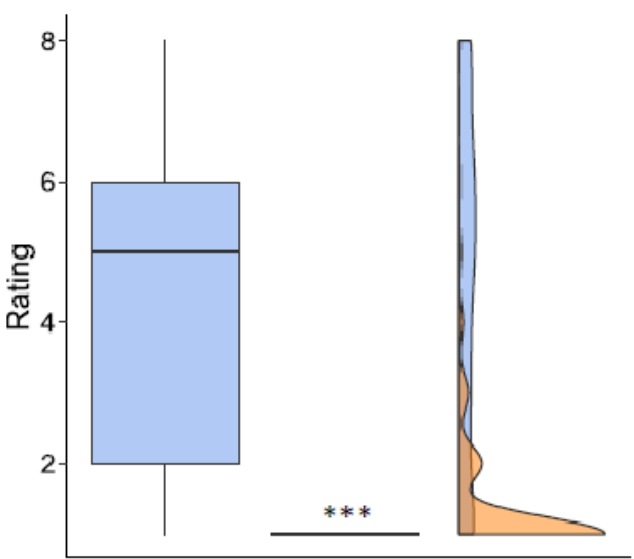

Empathy

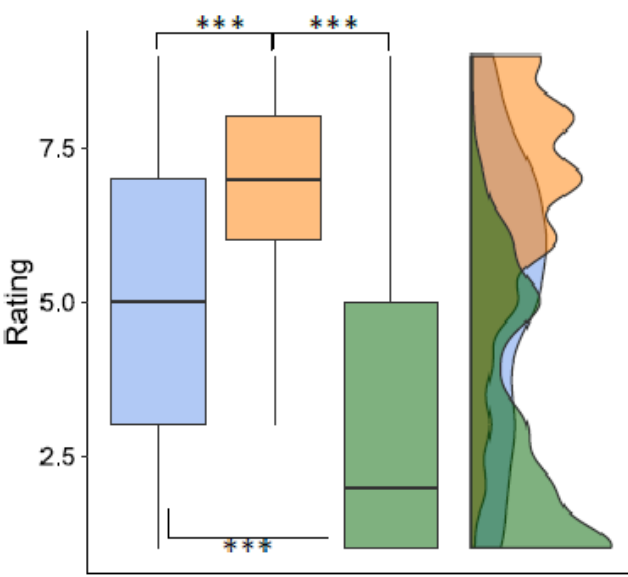

Help

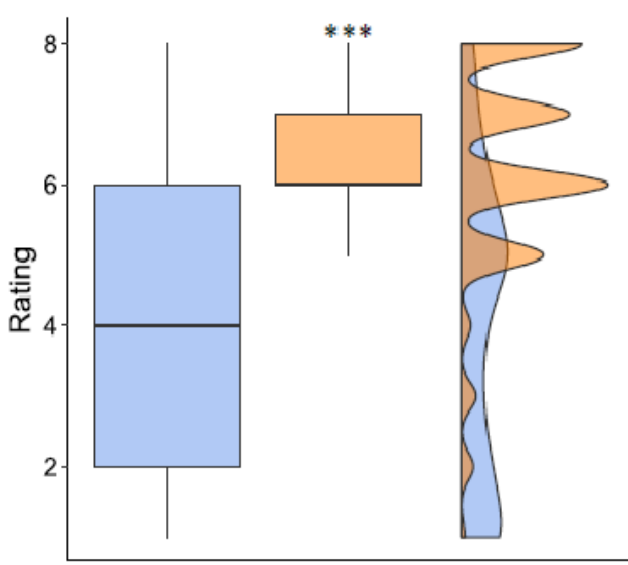

Figure 2. Effect of different context-video pairs on self-report measures. Box plots and half-violin plots displaying median values and distribution of responses to questions on valence, arousal, empathy (first row, scale values: 1 to 9 . For Valence, the scale is bipolar: $1=$ very negative, $5=$ neutral, $9=$ very positive) and responsibility, deservingness, willingness to help (second row, scale from 1 to 8 ). ${ }^{* * *}: \mathrm{p}<.001,{ }^{*}: \mathrm{p}<.01, *: p$ $<.05$ 


\subsection{Relationships between variables}

Our within-subject design allowed us to investigate the relationships between some of the variables implemented in the present study at the individual level and compare them to existing populationlevel models (Rudolph et al. 2004). Table 2 shows the correlations between all pairs of variables. It appears that valence correlates poorly with other variables, its strongest association being with arousal $(r(598)=-.18)$. Similarly, arousal is strongly associated only with empathy $(r(598)=.61)$. The relationships between responsibility, empathy and help are all in the expected directions. However, their magnitude differs from the literature (table 3 in Rudolph et al. 2004 meta-analysis). We found a moderate-to-strong relationship between responsibility and help $(r(480)=-.48,95 \% \mathrm{Cl}=-.42,-.55)$, whereas it is weak in the literature $(r(6838)=-.25,95 \% \mathrm{Cl}=-.23,-.27)$. Conversely, we found a weaker relationship between responsibility and empathy $(r(540)=-.24,95 \% \mathrm{Cl}=-.16,-.29)$ than the one between responsibility and sympathy in this literature $(r(7414)=-.45,95 \% \mathrm{Cl}=-.43,-.46)$.

Table 2. Correlations between self-report measures

\begin{tabular}{lccccccc}
\hline Variable & $\mathrm{n}$ & 1 & 2 & 3 & 4 & 5 & 6 \\
\hline 1. Valence & 600 & - & & & & \\
2. Arousal & 600 & $-.18^{* * *}$ & - & & & & \\
3. Empathy & 600 & $-.10^{*}$ & $.61^{* * *}$ & - & & & \\
4. Responsibility & 542 & -.05 & -.08 & $-.24^{* * *}$ & - & & \\
5. Deservingness & 512 & .07 & $-.15^{* * *}$ & $-.36^{* * *}$ & $.65^{* * *}$ & - & \\
6. Help & 482 & $-.11^{*}$ & $.26^{* * *}$ & $.50^{* * *}$ & $-.48^{* * *}$ & $-.62^{* * *}$ & - \\
\hline
\end{tabular}

${ }^{* * *} p<.001 * * p<.01^{*} p<.05$

Raw correlations are poorly informative for sets of intercorrelated variables. In order to obtain specific, unconfounded associations between variables, we used partial correlation as a measure of uniquely shared variance between pairs of variables. Figure 3 depicts the regularized partial 
correlation network obtained from within-subject centred data. We did not find any specific direct association between responsibility and help; instead, deservingness shared unique variance with both variables, consistent with a mediation role (similar to anger mediating the relationship between controllability and help giving in Rudolph et al. 2004). However, we did not find any significant specific relationship between empathy and responsibility. The negative result may have been due to secondary variables containing part of the unique variance shared between empathy and responsibility, or to empathy sharing similar (and therefore non-unique) variance with both responsibility and deservingness. To test this hypothesis, we computed the partial correlation between empathy and responsibility (or deservingness) after controlling only for the variance due to willingness to help. We found that none were significant $\left(R_{\text {emp-resp }}=.00, p>.96 ; R_{\text {emp-dsrv }}=-.09, p=.062\right)$.

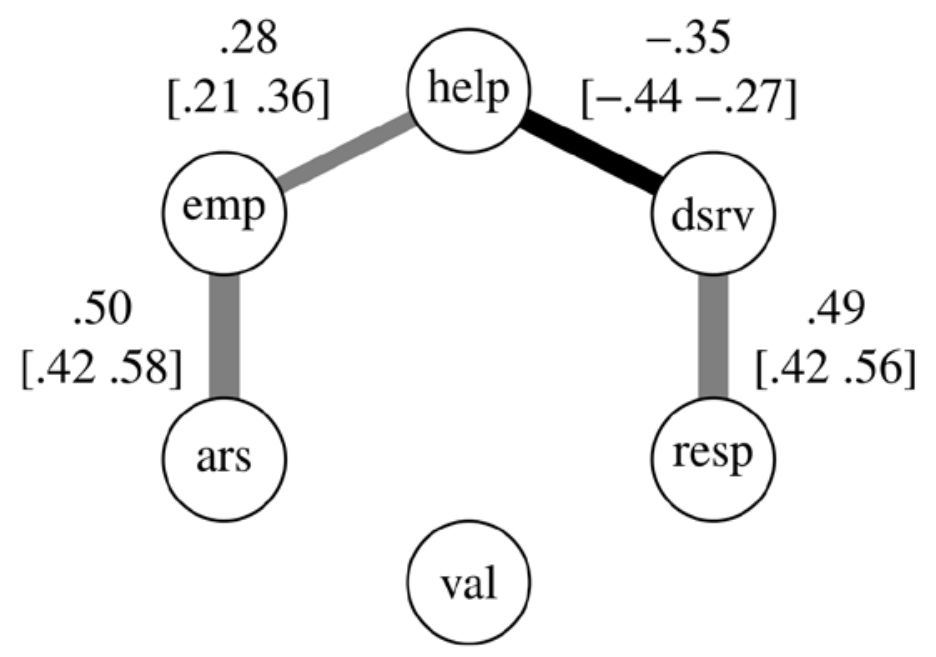

Figure 3. Regularized partial correlations network between within-subject centered variables. Edges represent partial correlations that survived a regularization step designed to avoid spurious associations without loss of power. Values indicate estimates and 95\% confidence intervals between brackets. VAL: valence, ARS: arousal, EMP: empathy, RESP: responsibility, DSRV: deservingness, HLP: willingness to help. Grey and black colours are associated, respectively, with positive and negative correlation values. 


\subsection{Effect of experimental manipulation on response times}

Figure 3 shows data distribution and significant contrasts for each scale.

For questions on valence, arousal and empathy, we found a statistically significant main effect of stimulus type (valence $F(2)=14.9, p<0.001$; arousal $F(2)=11.5, p<0.001$, empathy $F(2)=22.6, p<$ 0.001 .

Post-hoc tests showed how response times to neutral videos were significantly faster in questions on valence and arousal, compared to emotional videos preceded by responsible contexts (valence emotional_Resp. - neutral: t-ratio(49.8) $=4.6, p<0.001$, estimate $[95 \% \mathrm{Cl}]=0.2[0.1,0.3]$; arousal emotional_Resp. - neutral: $t$-ratio(51.8) $=4.2, p<0.001$, estimate $[95 \% \mathrm{Cl}]=0.2[0.1,0.2])$. For these questions, no difference in response times was found between neutral videos and emotional videos preceded by not responsible contexts (valence emotional_NotResp. - neutral: t-ratio(45.6) $=1.6, p=$ 0.27 , estimate $[95 \% \mathrm{Cl}]=0.06[-0.03,0.15]$; arousal emotional_NotResp. - neutral: t-ratio $(50)=1.7, p$ $=0.23$, estimate $[95 \% \mathrm{Cl}]=0.06[-0.03,0.15])$. Response times to empathy questions were faster for neutral videos compared to emotional videos preceded by responsible, as well as not responsible contexts (emotional_Resp. - neutral: t-ratio $(48.2)=6.2, p<0.001$, estimate $[95 \% \mathrm{Cl}]=0.24[0.14,0.33]$; emotional_notResp. - neutral: $t$-ratio $(51.1)=2.8, p=0.02$, estimate $[95 \% \mathrm{Cl}]=0.12[0.02,0.22])$

For all questions, response times to emotional videos preceded by responsible were significantly slower than those to not responsible contexts. A summary of the estimates and significance of posthoc tests for each scale is reported in Table 3. 


\begin{tabular}{|l|c|c|c|}
\cline { 2 - 4 } \multicolumn{1}{c|}{} & \multicolumn{3}{c|}{ Emotional_Resp. - Emotional_NotResp } \\
\hline Variable & t-ratio $(d f)$ & $p$ & estimate $(95 \%$ Cl) \\
\hline Valence & $4.2(14.7)$ & $\mathbf{0 . 0 0 2}$ & $0.1(0.04,0.17)$ \\
\hline Arousal & $3.5(15.1)$ & $\mathbf{0 . 0 0 8}$ & $0.09(0.02,0.15)$ \\
\hline Empathy & $4.1(15.5)$ & $\mathbf{0 . 0 0 2}$ & $0.12(0.05,0.2)$ \\
\hline Responsibility & $3.5(28.6)$ & $\mathbf{0 . 0 0 1}$ & $0.15(0.06,0.23)$ \\
\hline Deservingness & $7.6(26)$ & $<.001$ & $0.28(0.2,0.36)$ \\
\hline Help & $4.8(22.7)$ & $<.001$ & $0.17(0.1,0.24)$ \\
\hline
\end{tabular}

Table 3. Summary statistics for the pairwise comparisons of log-transformed response times estimated marginal means between responsible and not responsible contexts (Emotional_Resp. - Emotional_NotResp) for emotional videos. t-ratios and $p$ values are reported, together with marginal means contrast estimates and $95 \%$ confidence intervals for each response scale. Estimates in the logarithmic scale correspond to differences in response times between contexts of 400-600ms for the valence, arousal and empathy questions, and $800-1200 \mathrm{~ms}$ for the responsibility, deservingness and help questions. 
国Emotional_Resp 目Emotional_NotResp 目 ${ }^{\text {Neutral }}$
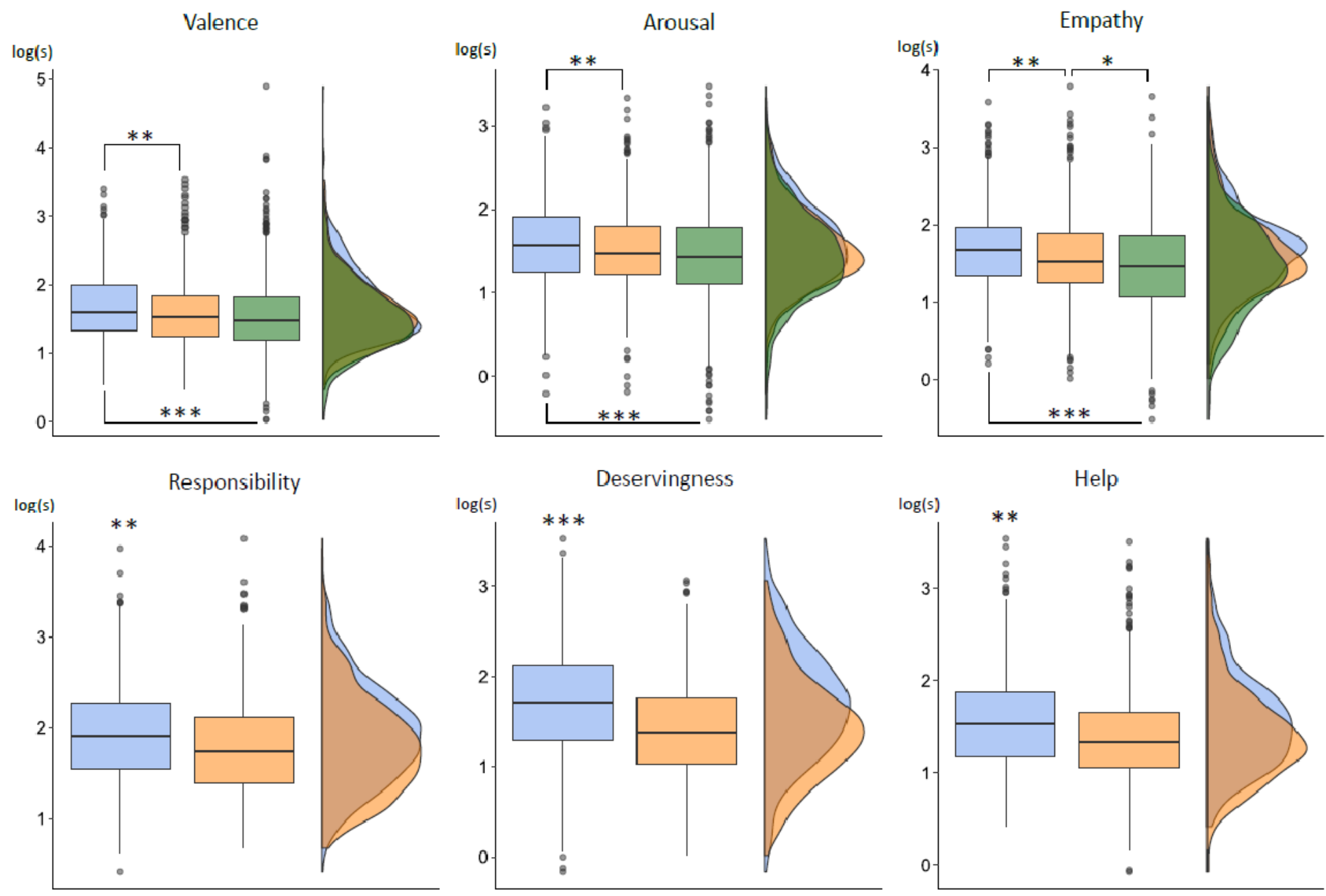

Figure 4. Effect of different context-video pairs on response times. Box plots and half-violin plots displaying median values and distribution of response log-transformed response times to questions on Valence, Arousal, Empathy (first row) and Responsibility, Deservingness, Willingness to help (second row). ${ }^{* * *}: \mathrm{p}<.001,{ }^{* *}: \mathrm{p}<$ $.01, *: p<.05$ 


\section{Discussion}

\subsection{Validation of stimuli}

In the present study, we created and validated a set of stimuli that can be implemented in experiments investigating the impact of attributional processes on responses to suffering and helping behaviour using within-subject designs and/or third person measures. Previous attempts in this area of study (e.g. Azevedo et al., 2014; Decety et al., 2009; Krendl et al., 2013) were affected by a number of limitations that impacted the generalizability and validity of the reported evidence. Here we took advantage of video stimuli that were previously validated along different affective dimensions and had already been implemented in neuroimaging experiments (Klimecki et al., 2013). On this basis, we created short stories for a subset of video stimuli, resulting in the manipulation of perceived responsibility in different scenarios

To validate the newly created set of stimuli, we investigated the effect of different context-video pairs on self-reported dimensions of subjective experience. The results of this analysis confirmed the capacity of different short stories preceding the same video to affect attributional processes. Participants' judgments on responsibility and deservingness of the sufferer(s) changed depending on the context associated with each video. Different contexts also modulated affective responses to emotional videos, as well as participants' willingness to help. The relation between these dimensions and the attribution of responsibility and deservingness was further explored and compared to previous studies on this topic (e.g. Rudolph et al., 2004; see section 4.2 for a discussion of the findings).

Different contexts also influenced the perceived valence of emotional videos. While we are not aware of previous evidence of the effect of responsibility attribution on perceived emotional valence, a topdown effect of context in emotional categorization has been described in several studies (see Brosch et al., 2010 for a review). In the present study, a significant effect of context was found, but the mean 
difference and the consistent overlap between confidence intervals of estimated marginal means point towards a very small effect size. Moreover, subsequent exploratory partial correlation analysis highlighted no link between valence and other scales. While this result should be further investigated when implementing this set of stimuli in future studies, it can be considered negligible in terms of its actual impact on the stimuli validation.

It is worth mentioning here that similar efforts have been recently made to provide standardized sets of stimuli to the research on Moral Foundations Theory (MFT, Graham et al., 2013). This line of research shares several questions with that on attributional processes, especially in terms of the interplay between cognition and affect in shaping moral judgment and helping behaviour. In this context, Clifford et al., (2015) validated a set of textual vignettes to address the limitations of currently employed stimuli for the study of MFT, which were affected by similar issues as the literature described in this manuscript (e.g. the lack of standardization and the focus on specific moral domains). In the present work, the use of video stimuli is sought to result in higher ecological validity and in the possibility of eliciting strong and clear affective responses (Grühn \& Sharifian, 2016), as well as to overcome limitations of the use of textual vignettes in blame attribution studies (Sleed et al., 2002). Based on these premises, we believe that the implementation of this validated set of context-video pairs can foster synergies between research fields such as the MFT and attributional theories of motivation (this aspect is further explored and discussed in section 4.3).

\subsection{Attributional processes at the within-person level}

Subsequently and complementary to the validation of stimuli, we sought to explore within-subject correlation patterns in our data, and compare them with established models derived from crosssectional data linking affective responses, attribution judgments and helping intentions. To this aim, we explored the relationships between the dimensions of subjective experience in response to emotional videos preceded by responsible contexts. 
Raw correlations showed a landscape of relationships between variables that is in line, albeit with some differences in terms of included dimensions and diverging magnitudes, with that previously reported by Rudolph et al. (2004) in a meta-analysis of studies on the effect of attributional processes on helping behaviour. Replicating previous studies, the present work highlights a negative relationship between willingness to help and attribution of responsibility. In addition, we found here a positive relationship between the degree of empathy for the sufferer and the willingness to help, as well as a strong association between attribution of responsibility and judgments on deservingness, which is in turn negatively correlated with willingness to help. The original attribution models include sympathy (intended as pity or compassion for the sufferer) instead of empathy (described here as to which degree a participant was moved by the sufferer's condition). Nonetheless, the correlation between empathy and willingness to help reported here is in line with the one between sympathy and help reported in Rudolph et al. (2004) meta-analysis. Similarly, attribution theory does not consider deservingness judgments as a relevant variable. However, the latter has been previously found to be associated with responsibility attribution (Kogut, 2011). Overall, this exploratory analysis replicates some of the previous results on the effect of attribution of responsibility and blame in modulating willingness to help and helping behaviour (Halkjelsvik \& Rise, 2014; Kogut, 2011; Rudolph et al., 2004). The results are also in line with the long-dated literature that describes a positive association between the degree of empathy and prosocial motivation (e.g. Batson et al., 1983, 1997; Eisenberg \& Miller, 1987).

The partial correlation network approach indicates that empathy and responsibility judgments act as independent, and possibly competing, factors in modulating helping intentions at the personal level. Similarly to established attribution models (Rudolph et al., 2004), there is no direct association between responsibility and willingness to help, which in this case appeared to be mediated by deservingness. Given that previous models do not include deservingness as a variable, the present results show that the assessment of moral judgments in the study of attribution processes could increase the understanding of mediating factors in prosocial motivation and compassion responses. 
Importantlywe did not find here an association between perceived responsibility and empathy. A negative relationship between responsibility and affective responses constitutes an important basis of attribution models (Rudolph et al., 2004). Here, the lack of correlation could be due to different reasons.

First, as previously mentioned, existing models assess the degree of sympathy instead of empathy. While sympathy is intended as feelings of concern for the other's suffering, here we assessed empathy as "the degree to which a participant was moved by the condition of the sufferer". Such discrepancy between the present and previous studies makes it difficult to compare results. However, the effect of different contexts on empathy and its relationship with willingness to help are in line with the one observed for sympathy in other studies.

Secondly, the inclusion of additional variables in the partial correlation analysis could have overshadowed a significant association as partial correlation models only highlight unique variance shared between variables. It is possible that, if both arousal and empathy were associated with responsibility, this non unique source of variance would be excluded from the model. However, this explanation is ruled out by the fact that the correlation value after partialling out only the variance due to willingness to help remains null.

Thirdly the correlation analysis was performed on a subset of data including only responsible context - emotional video pairs. The exclusion of not responsible contexts might have overshadowed a significant relationship between responsibility and empathy. However, the inclusion of those observations could lead to spurious correlations, due to the bimodal nature of the distributions of responses to the questions on responsibility and empathy when data is pooled across contexts (see figure 2). This issue could also affect evidence reported in previous studies, where path analysis was performed on the whole set of observations independently of the distribution of responses.

Excluding the explanations above, the discrepancies between the pattern of associations between variables presented here and classical models of the attributional literature could stem from the 
within-subject nature of our experimental design. This possibility warrants a better controlled and tighter comparison between results obtained with within-subject and inter-individual differences approaches.

\subsection{Attributional processes and responses times}

At last, we sought to demonstrate how implementing the newly created set of stimuli into repeatedmeasures experimental designs allows for the collection of implicit, third-person measures that could provide viable information on the behavioural correlates of decision-making, affective processes and moral judgment involved in the interplay between attribution and help. As an example, we explored the effect of the different context - video pairs on responses times for all self-report scales. Interestingly, for all questions following emotional videos, participants took longer to answer when the video was preceded by the Responsible, compared to the Not Responsible context. Given the exploratory nature of the analysis, we cannot provide a solid interpretation for this effect. Nonetheless, we can speculate on possible explanations and questions that arise from this evidence.

The longer response times could be due to a general increase in task demands, with more cognitive efforts required to answer questions for Responsible contexts. While this represents a simple interpretation, it is not clear which factors would underlie this effect. As fundamental stimuli characteristics remain unchanged, independently on the context type, longer response times can only derive from an effect on "internal" processes. In this sense, more refined interpretations can be suggested when considering evidence and theories that tap into the interplay between emotion and reasoning underlying reactions to morally loaded situations. This effect could, for instance, be caused by an incongruence between affective responses and task demands. In the context of studies on moral dilemmas, Greene et al., (2001) showed that judgments on moral decisions took longer when emotional responses conflicted with such decisions. In the present case, a general, and automatic, 
empathic response to suffering might conflict with the assessment of the situation in Responsible contexts, which remains purely imaginary and requires participants to effortfully interpret the "reality" of suffering displayed in the videos.

A third interpretation could be based on the interplay between affect and reasoning in moral judgments, as described in the context of the Social Intuitionist Model (SIM; Haidt, 2001). In brief, the SIM posits that moral judgments are generally made quickly and intuitively, while reasoning as an effortful process comes after and serves the purpose of finding arguments for the judgments that are already made (Sinnott-Armstrong, 2007). According to the SIM, moral reasoning is done merely to socially justify one's judgments. This position has been criticized by researchers who put emphasis on a more active role of reasoning in shaping moral judgments (e.g. Pizarro \& Bloom, 2003). Independently of the critiques, it seems plausible that attribution processes interact with the way moral judgments are made. In the present study, the effect of Responsible contexts on response times could be due to the engagement of more effortful reasoning, compared to Not Responsible contexts where a more intuitive appraisal of the situation is predominant.

Overall, this exploratory analysis provides evidence for an effect of attributional processes on implicit behavioural correlates. The questions that arise from the interpretation of these findings deserve further investigations. Most importantly, we showed here how new hypotheses and possible synergies between attribution accounts and prominent theories in moral psychology can be fostered by the implementation of stimuli that overcome the limitations of previous studies in this field.

\subsection{Limitations}

Here we describe limitations of the present study and issues that deserve further investigation. In our opinion, they mainly pertain to the characterization of stimuli, especially in terms of confounds that might affect aspects of their validation.

First, some context-video pairs might have introduced confounds that affect participants willingness to help beyond responsibility attribution. Along with responsibility, a person's likeability might have 
been manipulated in different contexts associated with the same emotional video (e.g. a terrorist compared to a teacher). Other confounds, such as in-group / out-group affiliation and whether a video depicts individuals or a group of sufferers, might have influenced responses to different videos.

Likeability can be considered as the most relevant confound as it differentially affects contexts associated with the same video. To tackle this, we attempted to mark those stimuli that may be affected by it (see the contexts file in the data repository at https://osf.io/8zytp/). On the other hand, the set of stimuli is characterized by a high degree of heterogeneity in terms of situations and characters, resulting in possibly few stimuli being affected by the issues described above. This view is corroborated by empirical data: in mixed effects models, the context variance between videos is smaller or similar to the one between participants, and this is true for all questions that should be impacted by likeability and other confounds (Responsibility, Deservingness, Willingness to Help; see table S1 at https://osf.io/8zytp/ for a detailed presentation of standard deviations for each random effect and each model).

In conclusion, while we cannot disentangle confounding effects from responsibility attribution for some videos and contexts, the set of stimuli is characterized by enough heterogeneity to make these confounds negligible. This is supported by the fact that, especially concerning willingness to help, the variation in responses is larger among subjects than among videos. Nonetheless, future studies implementing this set of stimuli could integrate measures of likeability (e.g. Wojciszke et al., 1993) to explicitly investigate the influence of this variable on willingness to help and its relationship with responsibility attribution.

A second issue affecting the present study is the lack of a proper control for the emotional intensity (arousal) of the video stimuli. Emotional videos depict situations characterized, on average, by high emotional arousal. They are compared to neutral videos, but this comparison is not relevant for those questions that investigate the effect of context on perceived responsibility, deservingness and willingness to help. As a result, we do not know whether the effect of context on emotional videos is 
generalisable across various degrees of emotional arousal. As this was not the original question of the present study, future research could integrate low-arousal video stimuli to the existing set and be able to address this question in a proper $2 \times 2$ design, including neutral stimuli as a baseline. On the other hand, the correlation analysis performed on the present dataset does not seem to suggest the presence of an interaction between emotional arousal and the effect of context. In particular, the partial correlation network did not highlight any significant relation between emotional arousal and responsibility attribution, deservingness or willingness to help in response to emotional videos.

The same issue is present when considering evaluative extremity. The present set of stimuli is characterized by highly negative emotional videos, often depicting extreme conditions. Even though the partial correlation analysis did not highlight any relationship between valence of emotional videos and responsibility attribution or willingness to help, future research could integrate moderately negative context-video pairs (e.g. somebody who fell off a bike while looking at their phone compared to somebody who fell due to an external agent) to investigate whether evaluative extremity interacts with responsibility attribution to determine helping intentions. On the other hand, extremely negative emotional videos are likely to elicit strong emotional responses. This can in turn facilitate the collection of neurophysiological data in within-subject experimental settings.

\section{Contributions}

$\mathrm{EF}, \mathrm{CB}$ and $\mathrm{AL}$ designed the stimuli and task; $\mathrm{EF}$ and JD collected data; $\mathrm{EF}, \mathrm{OA}$ and JD analysed data; $\mathrm{EF}$, $\mathrm{OA}, \mathrm{CB}$ and $\mathrm{AL}$ wrote the manuscript.

\section{Acknowledgements}

We thank Olga Klimecki for sharing the original set of video stimuli used with Tania Singer in the SoVT, from which the stimuli presented here are derived (Klimecki et al., 2013). We also thank Léa Longo and Lenny Roche for their help in preparing the stimuli. Author's contribution: 


\section{Funding information}

This study was supported by a European Research Council grant ERC-Consolidator 617739BRAINandMINDFULNESS to Antoine Lutz and by a Mind \& Life Institute Francisco J. Varela Research Award (2015) to Enrico Fucci.

\section{Competing interests}

The authors have no conflict of interests.

\section{Data accessibility statement}

All the stimuli and material used in the present study are publicly available, along with raw data and analysis scripts, at a repository of the Open Science Framework (https://osf.io/8zytp/).The manuscript contains detailed information that should allow a replication of the findings. If further information is needed, please contact the corresponding author. The experiment was not preregistered. When using this set of stimuli for new research, please credit the authors of Klimecki et al. (2013) in addition to the present study. 


\section{References}

Azevedo, R. T., Macaluso, E., Viola, V., Sani, G., \& Aglioti, S. M. (2014). Weighing the stigma of weight: An fMRI study of neural reactivity to the pain of obese individuals. Neurolmage, 91, 109-119. https://doi.org/10.1016/j.neuroimage.2013.11.041

Bates, D., Mächler, M., Bolker, B., \& Walker, S. (2014). Fitting linear mixed-effects models using Ime4. ArXiv Preprint ArXiv:1406.5823.

Batson, C. D., O'Quin, K., Fultz, J., \& Vanderplas, M. (1983). Influence of Self-Reported Distress and Empathy on Egoistic Versus Altruistic Motivation to Help. Journal of Personality and Social Psychology, 45(3), 706-718.

Batson, C. D., Sager, K., Garst, E., Kang, M., Rubchinsky, K., \& Dawson, K. (1997). Is Empathy-Induced Helping Due to Self-Other Merging? Journal of Personality and Social Psychology, 73(3), 495509.

Brosch, T., Pourtois, G., \& Sander, D. (2010). The perception and categorisation of emotional stimuli: A review. Cognition and Emotion, 24(3), 377-400. https://doi.org/10.1080/02699930902975754

Brysbaert, M. (2019). How many participants do we have to include in properly powered experiments? A tutorial of power analysis with reference tables. Journal of Cognition, 2(1), 16. https://doi.org/10.5334/joc.72

Clifford, S., Iyengar, V., Cabeza, R., \& Sinnott-Armstrong, W. (2015). Moral foundations vignettes: A standardized stimulus database of scenarios based on moral foundations theory. Behavior Research Methods, 47(4), 1178-1198. https://doi.org/10.3758/s13428-014-0551-2

Decety, J., Echols, S., \& Correll, J. (2009). The Blame Game: The Effect of Responsibility and Social Stigma on Empathy for Pain. Journal of Cognitive Neuroscience, 22(5), 985-997. https://doi.org/10.1162/jocn.2009.21266 
Eisenberg, N., \& Miller, P. A. (1987). The Relation of Empathy to Prosocial and Related Behaviors. Psychological Bulletin, 101(1), 91-119.

Epskamp, S., Borsboom, D., \& Fried, E. I. (2018). Estimating psychological networks and their accuracy: A tutorial paper. Behavior Research Methods, 50(1), 195-212. https://doi.org/10.3758/s13428-017-0862-1

Epskamp, S., Cramer, A. O. J., Waldorp, L. J., Schmittmann, V. D., \& Borsboom, D. (2012). qgraph: Network Visualizations of Relationships in Psychometric Data. Journal of Statistical Software, 48(4). https://doi.org/10.18637/jss.v048.i04

Goetz, J. L., Keltner, D., \& Simon-Thomas, E. (2010). Compassion: An evolutionary analysis and empirical review. Psychological Bulletin, 136(3), 351-374. https://doi.org/10.1037/a0018807

Graham, J., Haidt, J., Koleva, S., Motyl, M., Iyer, R., Wojcik, S. P., \& Ditto, P. H. (2013). Moral Foundations Theory: The Pragmatic Validity of Moral Pluralism. In P. Devine \& A. Plant (Eds.), Advances in Experimental Social Psychology (Vol. 47, pp. 55-130). Academic Press. https://doi.org/10.1016/B978-0-12-407236-7.00002-4

Greene, J. D., Sommerville, R. B., Nystrom, L. E., Darley, J. M., \& Cohen, J. D. (2001). An fMRI Investigation of Emotional Engagement in Moral Judgment. Science, 293(5537), 2105-2108. https://doi.org/10.1126/science.1062872

Grühn, D., \& Sharifian, N. (2016). Lists of Emotional Stimuli. In H. L. Meiselman (Ed.), Emotion Measurement (pp. 145-164). Woodhead Publishing. https://doi.org/10.1016/B978-0-08$100508-8.00007-2$

Haidt, J. (2001). The emotional dog and its rational tail: A social intuitionist approach to moral judgment. Psychological Review, 108(4), 814-834. https://doi.org/10.1037/0033295X.108.4.814

Halkjelsvik, T., \& Rise, J. (2014). Social Dominance Orientation, Right-Wing Authoritarianism, and Willingness to Help Addicted Individuals: The Role of Responsibility Judgments. Europe's Journal of Psychology, 10(1), 27-40. https://doi.org/10.5964/ejop.v10i1.669 
Hamaker, E. L. (2012). Why researchers should think "within-person": A paradigmatic rationale. In Handbook of research methods for studying daily life (pp. 43-61). The Guilford Press.

Holm, S. (1979). A Simple Sequentially Rejective Multiple Test Procedure. Scandinavian Journal of Statistics, 6(2), 65-70. JSTOR.

Klimecki, O. M., Leiberg, S., Lamm, C., \& Singer, T. (2013). Functional Neural Plasticity and Associated Changes in Positive Affect After Compassion Training. Cerebral Cortex, 23(7), 1552-1561. https://doi.org/10.1093/cercor/bhs142

Kogut, T. (2011). Someone to blame: When identifying a victim decreases helping. Journal of Experimental Social Psychology, 47(4), 748-755. https://doi.org/10.1016/j.jesp.2011.02.011

Krendl, A. C., Moran, J. M., \& Ambady, N. (2013). Does context matter in evaluations of stigmatized individuals? An fMRI study. Social Cognitive and Affective Neuroscience, 8(5), 602-608. https://doi.org/10.1093/scan/nss037

Lenth, R., Singmann, H., Love, J., Buerkner, P., \& Herve, M. (2020). emmeans: Estimated Marginal Means, aka Least-Squares Means (1.4.5) [Computer software]. https://CRAN.Rproject.org/package=emmeans

Mojtabai, R. (2010). Mental illness stigma and willingness to seek mental health care in the European Union. Social Psychiatry and Psychiatric Epidemiology, 45(7), 705-712. https://doi.org/10.1007/s00127-009-0109-2

Nakagawa, S., \& Schielzeth, H. (2013). A general and simple method for obtaining $R^{2}$ from generalized linear mixed-effects models. Methods in Ecology and Evolution, 4(2), 133-142. https://doi.org/10.1111/j.2041-210x.2012.00261.x

Peirce, J., Gray, J. R., Simpson, S., MacAskill, M., Höchenberger, R., Sogo, H., Kastman, E., \& Lindeløv, J. K. (2019). PsychoPy2: Experiments in behavior made easy. Behavior Research Methods, 51(1), 195-203. https://doi.org/10.3758/s13428-018-01193-y

Philip, J., Chadee, D., \& Yearwood, R. P. (2014). Health care students' reactions towards HIV patients: Examining prejudice, emotions, attribution of blame and willingness to interact with 
HIV/AIDS patients. AIDS Care, 26(10), 1236-1241.

https://doi.org/10.1080/09540121.2014.896449

Pizarro, D. A., \& Bloom, P. (2003). The intelligence of the moral intuitions: A comment on Haidt (2001). Psychological Review, 110(1), 193-196. https://doi.org/10.1037/0033-

295X.110.1.193

R core team. (2017). R: A language and environment for statistical computing. $R$ Foundation for Statistical Computing, Vienna, Austria. https://www.R-project.org/

Rudolph, U., Roesch, S., Greitemeyer, T., \& Weiner, B. (2004). A meta-analytic review of help giving and aggression from an attributional perspective: Contributions to a general theory of motivation. Cognition \& Emotion, 18(6), 815-848.

https://doi.org/10.1080/02699930341000248

Seacat, J. D., Hirschman, R., \& Mickelson, K. D. (2007). Attributions of HIV Onset Controllability, Emotional Reactions, and Helping Intentions: Implicit Effects of Victim Sexual Orientation. Journal of Applied Social Psychology, 37(7), 1442-1461. https://doi.org/10.1111/j.15591816.2007.00220.x

Sinnott-Armstrong, W. (Ed.). (2007). Social Intuitionists Answer Six Questions about Moral Psychology. In Moral Psychology. The MIT Press. https://doi.org/10.7551/mitpress/7573.003.0006

Sleed, M., Durrheim, K., Kriel, A., Solomon, V., \& Baxter, V. (2002). The Effectiveness of the Vignette Methodology: A Comparison of Written and Video Vignettes in Eliciting Responses about Date Rape. South African Journal of Psychology, 32(3), 21-28. https://doi.org/10.1177/008124630203200304

Vogel, D. L., Wade, N. G., \& Hackler, A. H. (2007). Perceived public stigma and the willingness to seek counseling: The mediating roles of self-stigma and attitudes toward counseling. Journal of Counseling Psychology, 54(1), 40-50. https://doi.org/10.1037/0022-0167.54.1.40 
Weber, M., Ziegele, M., \& Schnauber, A. (2013). Blaming the Victim: The Effects of Extraversion and Information Disclosure on Guilt Attributions in Cyberbullying. Cyberpsychology, Behavior, and Social Networking, 16(4), 254-259. https://doi.org/10.1089/cyber.2012.0328

Weiner, B. (2006). Social motivation, justice, and the moral emotions: An attributional approach. Lawrence Erlbaum Associates Publishers. https://doi.org/10.4324/9781410615749

Wojciszke, B., Brycz, H., \& Borkenau, P. (1993). Effects of Information Content and Evaluative Extremity on Positivity and Negativity Biases. Journal of Personality and Social Psychology, 64(3), 327-335. 